\title{
OBTENÇÃO DE NANOWHISKERS DE CELULOSE A PARTIR DOS RESÍDUOS DA HIDRÓLISE ENZIMÁTICA DO BAGAÇO DE CANA-DE-AÇUCAR
}

\author{
L. A. de CAMARGO ${ }^{1,2}$, S. C. PEREIRA ${ }^{2}$, C. S. FARINAS ${ }^{2,3}$, J. M. MARCONCINI ${ }^{2}$ e L. H. C. \\ MATTOSO $^{1,2}$ \\ ${ }^{1}$ Universidade Federal de São Carlos, Departamento de Química \\ ${ }^{2}$ Empresa Brasileira de Pesquisa Agropecuária, Embrapa Instrumentação \\ ${ }^{3}$ Universidade Federal de São Carlos, Departamento de Engenharia Química \\ E-mail: cristiane.farinas@embrapa.br
}

\begin{abstract}
RESUMO - O Brasil está progredindo quando se trata da substituição de combustíveis fósseis por etanol renovável obtido a partir da cana-de-açúcar. Devido às demandas ambientas e econômicas, a reutilização do resíduo da etapa de hidrólise enzimática da produção de etanol celulósico tem se tornado de grande relevância para garantir a viabilidade desse processo. Assim, o presente estudo avaliou o emprego dos resíduos da hidrólise enzimática do bagaço de cana-de-açúcar para a produção de nanowhiskers de celulose. O bagaço pré-tratado foi hidrolisado utilizando-se um extrato enzimático comercial $(10 \mathrm{mg}$ de proteína/g de celulose). A caracterização inicial do bagaço resultou em $61,5 \%$ de celulose, $4,5 \%$ de hemicelulose e $32,0 \%$ de lignina. A hidrólise enzimática do bagaço pré-tratado alcançou cerca de $60 \%$ de conversão, gerando um resíduo sólido de grande potencial para a produção de nanowhiskers de celulose, que é um material de alto valor agregado com aplicação em diversos setores.
\end{abstract}

\section{INTRODUÇÃO}

O setor de transporte costumava ser altamente dependente de combustíveis a base de petróleo, os quais acarretam altos impactos ambientais, principalmente relacionados ao efeito estufa. Além disso, a possibilidade de escassez das reservas de petróleo aliada à crescente preocupação com a preservação do meio ambiente são os principais motivadores da busca por alternativas para a produção de combustíveis sustentáveis. Neste cenário, o Brasil ocupa posição de destaque e está avançando quando se trata de substituir os combustíveis fósseis por bioetanol renovável. Devido a isto, desde 1925 o país vem utilizando este biocombustível, e sua produção teve um aumento de $70 \%$ em relação à produção e o consumo de gasolina (Balat, 2011).

Atualmente, uma potencial fonte para a produção de etanol é o bagaço de cana-de-açúcar. Neste caso, o bioproduto é denominado etanol de segunda geração ou etanol celulósico. O bagaço é um subproduto abundante e que contém teores elevados de carboidratos, sendo composto por 20-30\% de lignina, 40-45\% de celulose e 30-35\% de hemicelulose (Sun et al., 2004). Portanto, o bagaço de cana- 
de-açúcar trata-se de uma fonte de matéria-prima para processos ecologicamente corretos (Bhattacharya et al., 2008; Klemm et al., 2005).

A rota de produção de etanol celulósico é promissora e envolve basicamente três etapas, a saber: pré-tratamento da biomassa, hidrólise enzimática e fermentação alcoólica. Por se tratar de um material com elevada recalcitrância, torna-se necessário à utilização de pré-tratamentos adequados que viabilizem a ação das enzimas para a reação de degradação da celulose em glicose, que é um açúcar fermentescível pelas leveduras. É interessante notar que as enzimas atuam particularmente sobre a porção amorfa da celulose, ou seja, é esperado que o resíduo da hidrólise enzimática seja composto por uma porção de celulose cristalina. Devido às demandas ambientas e econômicas, a reutilização do resíduo da etapa de hidrólise enzimática da produção de etanol celulósico tem se tornado de grande relevância para garantir a viabilidade desse processo.

As cadeias poliméricas de celulose cristalina organizam-se nas microfibrilas das células vegetais em forma de nanocristais alongados e de pequenos diâmetros. Estes nanocristais são característicos de estruturas denominadas whiskers. Procedimentos que envolvam a digestão dos domínios amorfos das fibras vegetais são capazes de liberar estes nanocristais de celulose deixando-os mais susceptíveis a processos químicos subsequentes (Moràn, et. al. 2008).

Neste sentido, a utilização do resíduo do processo de degradação enzimática do bagaço de canade-açúcar para a produção de novos materiais, como nanowhiskers de celulose, para a utilização em diversos setores da indústria, entre eles, o de produção de embalagens, torna-se de grande relevância para a economicidade do processo global de produção de etanol celulósico em escala industrial. Com esta motivação, o presente trabalho avaliou a viabilidade de reutilização do resíduo da hidrólise enzimática do bagaço de cana-de-açúcar pré-tratado por explosão a vapor para a produção de nanowiskers de celulose.

\section{MATERIAIS E MÉTODOS}

\subsection{Materiais}

Os materiais utilizados foram o bagaço de cana-de-açúcar in natura (BIN) e o bagaço de canade-açúcar pré-tratado por explosão a vapor (BEX) (Centro de Tecnologia Canavieira). O complexo enzimático comercial Cellic ${ }^{\circledR} \mathrm{CTec} 2$ (Novozymes) foi usado na etapa de hidrólise enzimática. No branqueamento do resíduo da hidrólise enzimática (RHE) foi utilizado o peróxido de hidrogênio $\left(\mathrm{H}_{2} \mathrm{O}_{2}\right) 34 \%$ e hidróxido de sódio $(\mathrm{NaOH}) 5 \%$.

\subsection{Métodos}

Preparação da biomassa: Primeiramente, o bagaço de cana pré-tratado por explosão a vapor (BEX) foi exaustivamente lavado e depois secado em estufa a $45{ }^{\circ} \mathrm{C}$ durante 48 horas. Por fim, procedeu-se a moagem em moinho de facas com granulometria inferior a $2 \mathrm{~mm}$. 


\section{9 a 22 de outubro de 2014 \\ Florianópolis/SC}

Caracterização química e morfológica: A composição química das amostras foi determinada de acordo com Gouveia et al. (2009). O índice de cristalinidade (IC \%) foi calculado com base no método de Segal et al. (1959), usando-se a Difração de Raio X (DR-X) em um equipamento Shimadzu 6000 com radiação $\mathrm{CuKa}(\lambda=1,54 \hat{\mathrm{A}})$. A identificação de grupos funcionais foi realizada por Infravermelho com Transformada de Fourier (FTIR) em um equipamento Perkin Elmer Spectrum One empregando-se um disco de $\mathrm{KBr}$ com $1 \%$ de amostra finamente moída. As análises microscópicas foram realizadas por Microscopia Eletrônica de Varredura (MEV) equipado com um canhão de emissão de campo (FEG). Para as análises por MEV, as amostras foram previamente fixadas em stubs de alumínio com fita de carbono e revestido com ouro (Sistema de Revestimento BAL -TEC MED 020).

Hidrólise enzimática: O bagaço de cana pré-tratado (BEX) foi submetido à hidrólise enzimática em tampão citrato $0,1 \mathrm{M}, \mathrm{pH} 5,0$ com uma carga de sólidos de $10 \%$. Utilizou-se $10 \mathrm{mg}$ de proteína por grama de celulose. A atividade enzimática foi previamente determinada de acordo com Ghose (1987). A concentração de proteínas em $\mathrm{mg} / \mathrm{mL}$ foi determinada pelo método de Bradford. Os ensaios foram realizados a $50^{\circ} \mathrm{C}$ e $200 \mathrm{rpm}$ durante $24 \mathrm{~h}$. A produção de glicose foi monitorada por um kit enzimático (Doles, Brasil).

Branqueamento do resíduo: A etapa de branqueamento seguiu a metodologia de Teixeira et al. (2011). Adicionou-se $100 \mathrm{~mL}$ de $\mathrm{NaOH}$ a $55^{\circ} \mathrm{C}$. Em seguida, adicionou-se $5 \mathrm{~g}$ do resíduo da hidrólise enzimática (RHE) e $43 \mathrm{~mL}$ de $\mathrm{H}_{2} \mathrm{O}_{2} 35 \%$ sob agitação magnética por 1,5 horas. A solução foi filtrada a vácuo em funil de Buchner lavando-se o resíduo até $\mathrm{pH}$ neutro. Este material foi seco a $70{ }^{\circ} \mathrm{C} \mathrm{em}$ estufa para as análises de caracterização.

\section{RESULTADOS E DISCUSSÃO}

A Tabela 1 apresenta os teores de celulose, hemicelulose e lignina. Pode-se observar que o prétratamento por explosão de vapor resultou num enriquecimento da biomassa em celulose, principalmente através da remoção da fração hemicelulósica. Depois do processo de hidrólise enzimática é possível notar que no resíduo houve uma diminuição da quantidade de celulose e um enriquecimento de lignina, resultado da degradação de uma porção da celulose por parte das enzimas. Cálculos preliminares haviam mostrado que a hidrólise enzimática do bagaço de cana-de-açúcar prétratado alcançou cerca de $60 \%$ de conversão gerando um resíduo composto por um teor de celulose residual, provavelmente a porção cristalina deste carboidrato, conforme pode ser confirmado pelos resultados apresentados na Tabela 1 e pelo índice de cristalinidade apresentado na Tabela 2.

Tabela 1 - Composição química do material lignocelulósico (\%)

\begin{tabular}{|cccc|}
\hline & Celulose (\%) & Hemicelulose (\%) & Lignina (\%) \\
\hline $\begin{array}{c}\text { Sem tratamento (BIN) } \\
\text { Pré-tratado Explosão a Vapor }\end{array}$ & 46,6 & 26,5 & 21,7 \\
$\begin{array}{c}\text { (BEX) } \\
\text { Resíduo da Hidrólise (RHE) }\end{array}$ & 61,5 & 4,5 & 32,0 \\
\hline
\end{tabular}




\section{9 a 22 de outubro de 2014 \\ Florianópolis/SC}

A Figura 1A mostra que os espectros FTIR do BEX e RHE foram semelhantes ao do BIN sugerindo que estes procedimentos não provocaram alterações drásticas em sua estrutura química. Tanto para o BIN quanto para o BEX e o RHE, observa-se uma banda em $3386 \mathrm{~cm}^{-1}$ característica de grupos $\mathrm{OH}$, além de uma banda em $2852 \mathrm{~cm}^{-1}$ que é atribuída a grupos $\mathrm{OCH}_{3}$; ambos estão geralmente presentes na estrutura da lignina. A observação de bandas em 3386 e $2852 \mathrm{~cm}^{-1}$ indica que houve uma conversão de grupos $\mathrm{OCH}_{3}$ da lignina para grupos fenólicos devido à explosão a vapor. $\mathrm{A}$ banda em $2920 \mathrm{~cm}^{-1}$ é atribuída a grupos $\mathrm{CH}_{2}$ e $\mathrm{CH}_{3}$ da celulose, lignina e hemicelulose. A banda $1735 \mathrm{~cm}^{-1}$ é referida a grupos acetil presentes na hemicelulose e podemos notar que este foi reduzido no espectro do BEX e volta a aparecer no espectro de RHE. As bandas a $1633 \mathrm{~cm}^{-1} \mathrm{e} 1604 \mathrm{~cm}^{-1}$ para o BIN e $1602 \mathrm{~cm}^{-1}$ para o RHE, são atribuídas à estrutura aromática de lignina. As bandas em 1424, 1380 e $1325 \mathrm{~cm}^{-1}$ são atribuíveis à celulose cristalina, enquanto a banda de $902 \mathrm{~cm}^{-1}$ no BEX e 894 $\mathrm{cm}^{-1}$ no RHE são atribuídas à celulose amorfa. A intensificação de $1425-1325 \mathrm{~cm}^{-1}$ do BIN para $o$ BEX corresponde a uma concentração de celulose cristalina.

Observando a Figura 1B, a qual apresenta o DR-X para as amostra de BEX, RHE e para o resíduo da hidrólise enzimática após o branqueamento (RHEB), é possível notar que houve uma diminuição na cristalinidade da amostra de BEX para a de RHE, onde as bandas de absorção se tornam menos intensas. Analisando-se os índices de cristalinidade (IC \%), que estão apresentados na Tabela 2, verifica-se que este passa de $65,0 \%$ no BEX para 54,0\% no RHE, confirmando o observado nos difratogramas. Esta diminuição ocorre devido à alta concentração de lignina (substância amorfa) encontrada no resíduo após a hidrólise enzimática. O DR-X do BIN (espectros não mostrados) apresentou um IC\% de 55,0\%, e após o tratamento por explosão a vapor, este índice foi elevado para $65,0 \%$, o qual pode ser elucidado pela remoção de hemicelulose (substância amorfa) a partir deste pré-tratamento.

(A)

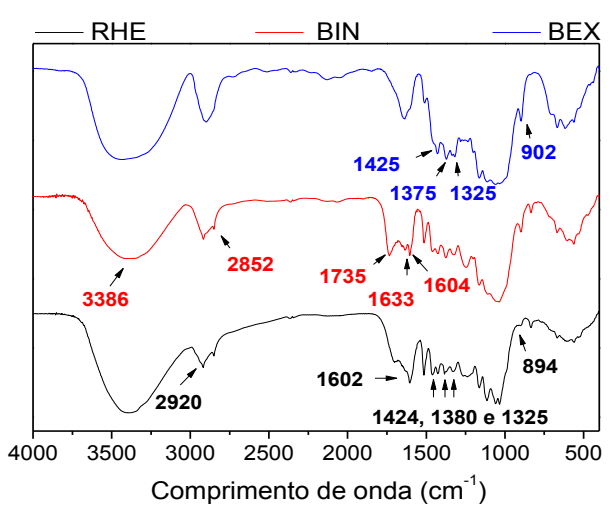

(B)

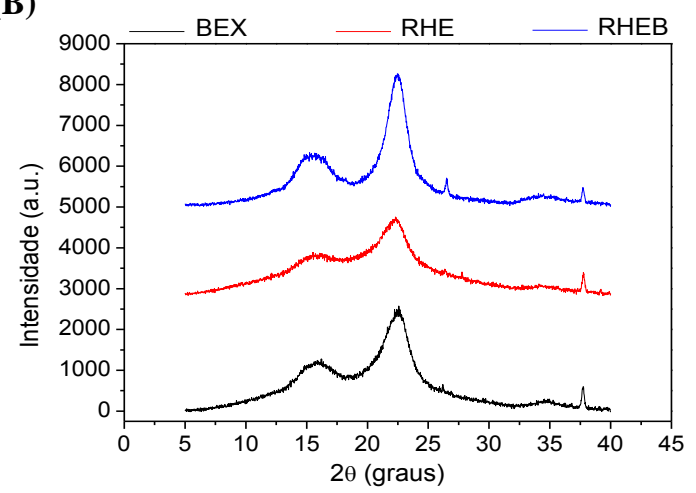

Figura 1 - Análises morfológicas do bagaço de cana: (A) espectros FTIR; (B) Difratograma de Raio$\mathrm{X}$ para o BEX, RHE e RHEB.

Após o branqueamento podemos ver no difratograma do RHEB que ocorreu novamente um aumento da cristalinidade devido ao alto índice de remoção da lignina. O IC \% para esta amostra foi de $83,2 \%$, o que esta de acordo com o verificado no difratogramas apresentado na Figura 1B e o esperado para este tipo de tratamento, conforme pode ser visto e confirmado pelas Tabelas 1 e 2 . Este 
alto índice de cristalinidade indica que grande parte da celulose residual após os processos de hidrólise enzimática e branqueamento está sob a forma do tipo cristalina, viabilizando o processo de produção de nanowhiskers de celulose a partir destas amostras.

Tabela 2 - IC (\%) para o BEX, RHE e RHEB

\begin{tabular}{|cccc|}
\hline & BEX (\%) & RHE (\%) & RHEB (\%) \\
\hline IC (\%) & 65,0 & 54,0 & 83,2 \\
\hline
\end{tabular}

Para completar os dados de caracterização, as micrografias foram obtidas por MEV. BIN (Figura 2A) apresenta uma morfologia rígida e compactada, enquanto o BEX e o RHE (Figura 2B e 2C) exibiram uma morfologia mais desorganizada, com uma maior exposição das fibras.
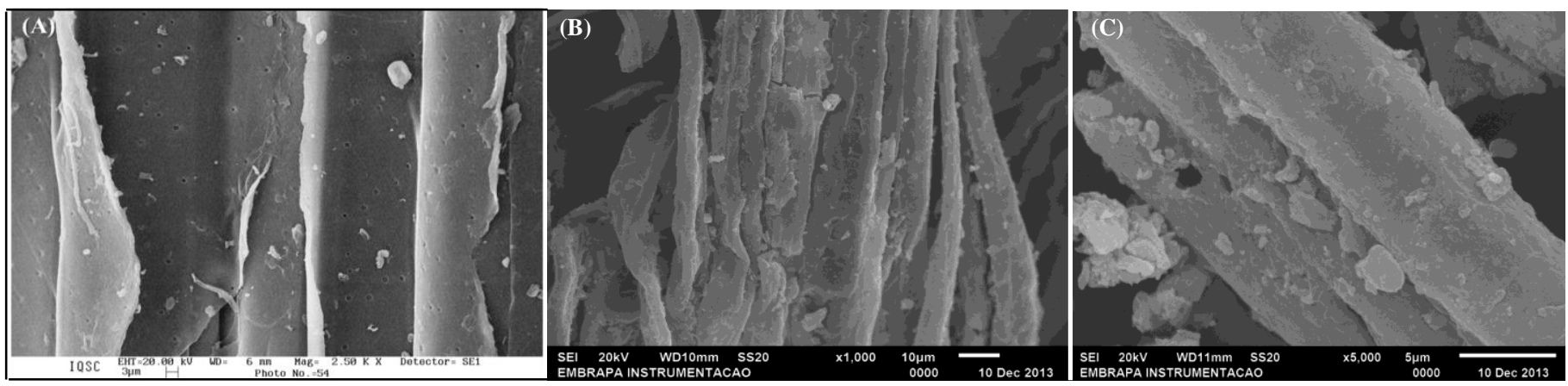

Figura 2 - Micrografia de MEV do bagaço de cana: (A) BIN (B) BEX (C) RHE.

\section{CONCLUSÃO}

A partir dos resultados apresentados neste trabalho, foi possível avaliar o mecanismo envolvido no pré-tratamento por explosão a vapor, bem como no procedimento de hidrólise enzimática e no branqueamento das fibras de bagaço de cana-de-açúcar. O procedimento de explosão a vapor mostrou-se eficaz no que diz respeito à remoção da fração hemicelulósica, o que contribuiu para a conversão de celulose a glicose. A hidrólise enzimática apresentou um fator de conversão de aproximadamente $60 \%$ nas condições avaliadas, gerando um resíduo sólido de grande potencial devido à quantidade de celulose cristalina presente no material. O branqueamento do resíduo possibilitou a remoção da lignina residual, permitindo uma maior exposição de celulose, sendo que este material apresentou um índice de cristalinidade de 83,2\%. Este índice mostra que este resíduo sólido apresenta grande potencial para a produção de nanowhiskers de celulose, o qual é um material de alto valor agregado com aplicação em diversos setores. Sendo assim, a utilização dos resíduos do processo de degradação para a produção de novos materiais como nanowhiskers de celulose para a utilização em diversas áreas da indústria, entre elas a de embalagens, faz com que a rota de hidrólise enzimática apresente características de interesse para utilização em grande escala. 


\section{NOMENCLATURA}

BEX - Bagaço de cana-de-açúcar pré-tratado por explosão a vapor

BIN - Bagaço de cana-de-açúcar in natura

IC (\%) - Índice de cristalinidade em porcentagem

RHE - Resíduo da hidrólise enzimática

RHEB - Resíduo da hidrólise enzimática após o branqueamento

\section{REFERÊNCIAS}

BALAT, M. Production of bioethanol from lignocellulosic materials via the biochemical pathway: A review. Energy Convers. Manage., 52,858-875, 2011.

BHATTACHARYA, D.; GERMINARIO, L. T.; WINTER, W. T. Isolation, preparation and characterization of cellulose microfibers obtained from bagasse. Carbohydr. Polym. 73, 371-377, 2008.

GHOSE, T.K. Measurement of cellulase activities. Pure Appl. Chem., v. 59, p. 257-268, 1987.

GOUVEIA, E.R; NASCIMENTO, R.T; SOUTO-MAIOR, A.M. Validação de metodologia para a caracterização química de bagaço de cana-de-açúcar. Quim. Nova, v. 32, p. 1500-1503, 2009.

KLEMM, D.; HEUBLEIN, B.; FINK, H.-P.; BOHN, A. Cellulose: Fascinating Biopolymer and

Sustainable Raw Material. Angew. Chem. Int. Ed., 44, 3358 - 3393, 2005.

MORÁN, J. I.; ALVAREZ, V. A.; CYRAS, V. P. \&VÁZQUEz, A. Extraction of celulose and preparation of nanocellulose from sisal fibers. Cellulose, 15, p.149,2008.

SEGAL, L.; CREELY, J.J.; MARTIN, A.E.; CONRAD, C.M. An empirical method for estimating the degree of crystallinity of native cellulose using the X-ray diffractometer. Text. Res. J., v. 29, p. 786-794, 1959.

SUN, J.X.; SUN, X. F.; ZHAO, H.; SUN, R.C.; Isolation and characterization of cellulose from sugarcane bagasse. Polym. Degrad. Stab. 84, 331 e 339, 2004.

TEIXEIRA, E. M.; BONDANCIA, T. J.; TEODORO, K. B. R.; CORREA, A. C.; MARCONCINI, J. M.; MATTOSO, L. H. C. Sugarcane bagasse whiskers: Extraction and characterizations. Ind. Crop. Prod. 33, 63-66, 2011. 